\title{
Bisphosphonate-related osteonecrosis of the jaw in a 66-year-old female - Case report
}

\author{
Anna Gaweda', Remigiusz Czerkies' ${ }^{1}$ Eliza Trzaskowska', Tomasz Tomaszewski' \\ ${ }^{1}$ Department of Maxillofacial Surgery. Medical University of Lublin
}

Gaweda A, Czerkies R, Trzaskowska E, Tomaszewski T. Bisphosphonate-related osteonecrosis of the jaw in a 66-year-old female - Case report. J Pre-Clin Clin Res. 2017; 11(2): 162-166. doi: 10.26444/jpccr/80726

\begin{abstract}
Bisphosphonate-related osteonecrosis of the jaws is an increasingly common side-effect of bisphosphonate treatment of malignant cancer of the osseous tissue. Necrotic foci of the bone with secondary inflammation are typical of the condition. The treatment is symptomatic and depends on the severity of the disease. The case is presented of BRONJ in a 66-year-old, occupationally-active female patient who had undergone implant-prosthetic treatment, and whose quality of life deteriorated significantly due to the symptoms of the disease. Bisphosphonate-related osteonecrosis of the jaw is treated conservatively at the initial stages of the disease, and surgical treatment should be avoided. Thorough oral hygiene and irrigations with chlorhexidine solution, supported by guided antibiotic therapy, are recommended. In severe cases, surgical sequestrotomy of the inflammatory foci, aimed at inhibiting or decelerating the course of the disease, is necessary.
\end{abstract}

I Key words

BRONJ, MRONJ, biphosphonates, impl antoprosthetic treatment, surgical debridement

\section{INTRODUCTION}

Bisphosphonates (BPs) are medications that inhibit osteoclasts activity and thus bone resorption and recreation. The most significant indications for BPs therapy include improving bone density in patients with osteoporosis, and reducing intraosseous development of primary malignant tumour or its metastasis. The drugs reduce hypercalcaemia in patients with primary bone tumours, such as multiple myeloma, as well as bone metastases of breast and prostate cancer $[1,2$, $3,4,5,6]$.

BPs are used in the prevention and treatment of osteoporosis, osteitis deformans, bone metastases of malignant cancer and primary tumours of the bone [4]. The medications slow down the remodeling process and increase bone mineral density by suppressing osteoclast activity, thereby reducing the risk of fracture in women with osteopenia and osteoporosis. All bisphosphonates reduce fractures of the spine by approximately $50 \%$ (Alendronate) and the hip by $30 \%$ (Risedronate) [4].

BPs do not affect bone formation directly through reductions in resorption. Bone formation may seem normal, but at the time of histological assessment the osteoid was no longer being formed. Since jaw bones have frequent microdamage and reconstruction, BP-related osteonecrosis occurs mostly in this region $[4,7]$. There are many theories about the causes of osteonecrosis solely on the jaws and not in other bones. Some review articles highlight the connection with excessive suppression of the jaw bone turnover, infection, angiogenesis inhibition, soft tissue toxicity, the immune system performance and microfractures $[8,3]$. The half-life of bisphosphonates (BPs) in bone is very long, ranging from 1 10 years, depending on the rate of bone turnover [8]. During remodeling of the jaws bone process, BPs accumulate in the bone. Other aspects have been reported, such as soft tissue

Adress for correspondence: Anna Gaweda, Department of Maxillofacial Surgery. Medical University of Lublin

E-mail: agaweda5@wp.pl

Received: 9 August 2017; accepted: 21 September 2017 toxicity leading to micro-abscesses and intense inflammatory infiltration in hypoderm, permeating to the muscle fibres and fat lobules [9].

Some patients treated with BPs develop non-healing or poorly healing osteonecrosis of the jaws. Necrotic fields increase and develop secondary infection, severe pain, pathologic fracture or resection of the bone $[1,6,4]$. Some patients present clinical symptoms of BRONJ, e.g. abscess, mucosal/gingival fistula, non-healing postextraction socket, swelling or trismus $[5,4]$. The disease is progressive and lasts for a long time during which the patients are treated as routine dental gingival problems resulting in deterioration of the quality of life $[5,3]$.

Histologically, several tissue alternations are observed in BRONJ, including necrotic bone honeycombed with residual vital bone, inflammatory cellular elements and hipernucleated osteoclasts and fibrous tissues [1].

The term Bisphosphonate Related Osteonecrosis of the Jaws (BRONJ) was changed in 2014 by the American Association of Oral and Maxillofacial Surgeons (AAOMS) to Medication Related Osteonecrosis of the Jaws (MRONJ), due to the increasing number of necrosis associated with antiresorptive and antiangiogenic medicaments different from bisphosphonates $[10,11]$. This condition can be diagnosed in a patient who presents the following characteristics:

1. Current or previous treatment with antiresorptive or antiangiogenic agents.

2. Exposed bone or fistula penetrating through the bone that had persisted for more than eight weeks.

3. No history of radiation therapy to the jaws or obvious metastatic disease to the jaws.

Medications associated with MRONJ include:

- antiresorptive agents, e.g. intravenous (more common) or oral bisphosphonates and RANK ligand inhibitor (denosumab);

- antiangiogenic agents (tyrosine kinase inhibitors and monoclonal antibody targeting VEGF) $[10,11,3,4,2]$. 
MRONJ can develop spontaneously or as a result of surgical treatment in the region of the jaws in cancer patients. In most cases, osteonecrosis of the jaws is related to administration of large quantities of bisphosphonates intravenously and prolonged exposure to the medications [4]. Surgical procedures, such as dental extractions, alveolar process implantation, periodontal surgery, periodontal inflammation, advanced age of patients, as well as concomitant cancer, favour MRONJ development. Favourable factors for necrosis is the presence of a surrounding thin mucous membrane, convexities and exostoses of the jaws. [3, 12].

Additional corticosteroid and immunosuppressive therapy in patients with impaired immune system due to malignant process favours the risk of MRONJ. General loads which additionally decrease the immunity of the jaw bones include diabetes, smoking cigarettes, drinking alcohol, poor oral hygiene $[1,3,4,9,12]$.

Current staging and treatment guideline. Patients at risk have no symptoms but they had intravenous or oral antiresorptive or antiangiogenic therapy. Patients at risk of BRONJ do not require treatment but have to be informed about the probability of the disease development:

Stage 0 - no exposed bone but symptoms like odontalgia or pain without clinical/radiological causes.

Stage I - exposed or necrotic bone in asymptomatic patients, with no evidence of infection. Due to pain, analgesics, antiinflammatory irrigations and antibiotic therapy (penicillin, fluoroquinolone, metronidazole, clindamycin, doxycycline, erythromycin) are required. Superficial inflammatory dermatoses review is applied. Surgical interventions needs to be avoided.

Stage II - exposed and/or necrotic bone with evidence of infection in symptomatic patients. Anti-inflammatory irrigations, antibiotic and analgesic therapy is required. Simple surgical revision of the involved area can be considered.

Stage III - exposed and/or necrotic bone with evidence of infection in symptomatic patients with destruction progressing to the adjacent area. The treatment involves surgical debridement, resection with antibiotic and analgesic therapy $[10,11,3,4]$.

Elective surgery of the alveolar process is not contraindicated in the course of oral BPs treatment, but the risk of osteonecrosis increases after a three-year treatment with the medications [3, 4]. BRONJ incidence rate in patients treated with medications administered intravenously is $2-18 \%$ [6], $0.8-18.5 \%$ [5], up to $21.8 \%$ [3], whereas the BRONJ incidence rate in the case of oral administration of the medication ranges from $0.01-0.04 \%$ [3]. Prevention is recommended for the patients from BRONJ risk groups. With the exception of cases where the onset of the disease is spontaneous, in some patients surgical procedures of the jaws should be eliminated. Prior to the implementation of BPs therapy, gangrenous teeth should be extracted and other surgical procedures of the jaws should be performed. Patients on oral BPs and corticosteroids who require surgical procedure in the oral cavity need to discontinue BPs at least three months before and after the procedure. This management significantly reduces the risk of osteonecrosis of the jaws [4].

Treatment of BRONJ involves local removal of the necrotic bone, analgesic and anti-inflammatory therapy, frequent antiseptic irrigations (0.12\% chlorhexidine) and discontinuation of BPs therapy. Surgical revision of the bone and removal of loose sequesters can reduce inflammatory foci, whereas too intense surgical intervention can cause intensification of the inflammatory condition. Therefore, during the procedure, reaching the margin of the healthy, bleeding bone is not recommended. Sharp edges of the bone, which can be continually irritated, should be extracted or ground without excessive interference in the surrounding bone tissue $[3,4]$. Patients with pathologic mandibular fracture qualify for segmental resection and stabilization with a reconstruction plate. In some cases, discontinuation of BPs therapy results in spontaneous sequestrotomy, clearance of the inflammatory focus, and thus improvement of the clinical condition $[3,4]$.

\section{CASE REPORT}

In 2015, a 66-year-old female was referred to our hospital. She had had implantoprosthetic treatment since 2006, including left lateral window approach sinus lift using BioOss material and a membrane. Implantation was performed in positions $15,14,17,23,24$ and 25 , as well as in 34,41 and 44 (Figs. 1-2). After the titanium implants healed, the patient had dentures made three times, initially supported onto the implants and after losing them, she had partial dentures.

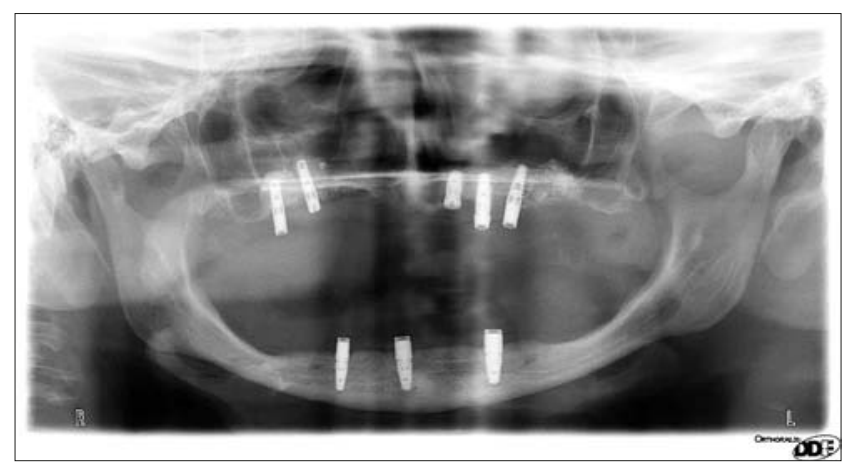

Figure 1. Implantation of the jaw and mandible. Panoramic radiograph after the implant in position No. 14 was lost, visible implants 15, 17, 23, 24, 25 and 34, 41, 44.

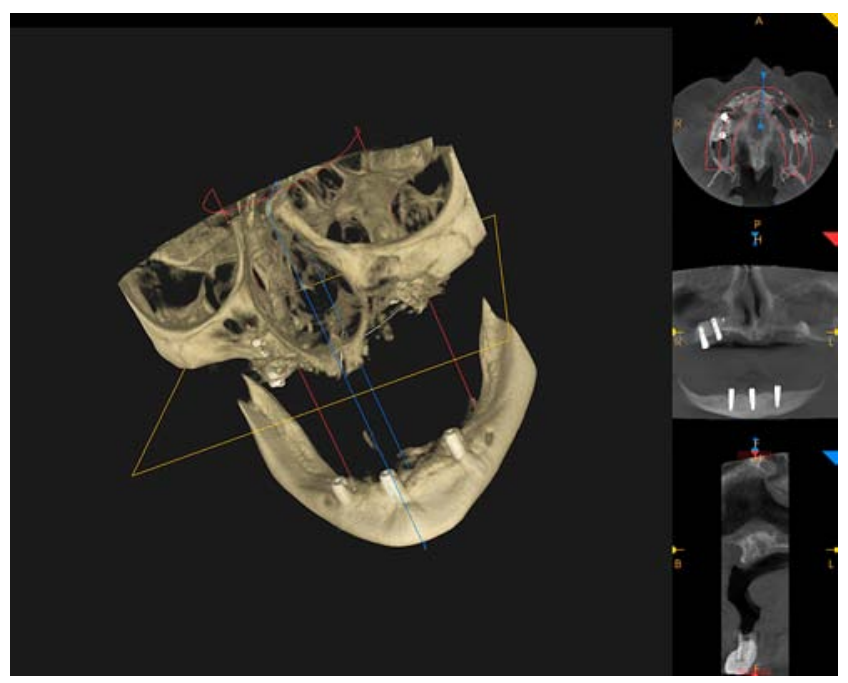

Figure 2. 3D CT reconstruction - condition after the loss of implant in positionsw 23,24 and 25 . Osteonecrotic foci of the right and left side of the jaw (white arrows), present implants 14, 15 (maxilla), 34, 41, 44 (mandible) 
In 2012, the patient underwent radiotherapy and chemotherapy due to breast cancer; she then took bisphosphonates intravenously for 3 years, initially Zoledronic Acid and next Pomitor, for approximately 5 months. After taking bisphosphonates for 1.5 years, the bone of the alveolar process was gradually exposed bilaterally and several inflammatory bone foci occurred.

The infectious process of the jaws originated from the periimplant regions and led to spontaneous loss of implants in the maxilla and mandible. The pathologic lesions in the jaw were evaluated as grade II and III. In September 2015, the patient was admitted to the Clinic of Maxillofacial Surgery of the Medical University in Lublin, Poland, for revision and sequestrotomy of the jaw bone.

Clinical examination revealed exposed, necrotic bone with evidence of infection bilaterally in the canine fossa area, communication with both maxillary sinuses, the mucosal swelling with severe pain, lack of maxillary implants (Fig. 3), presence of two mandibular implants in positions 33, 44 (Fig. 4), epiphora and fetor ex ore. Results of standard laboratory tests were within normal ranges. Chest X-ray revealed numerous speckled osteolytic defects in the ribs and clavicles projection, sclerosis of the vertebral bodies L2 and L1 with a compression fracture; fracture of the side section of the sixth and eighth right rib suggested metastatic lesions.

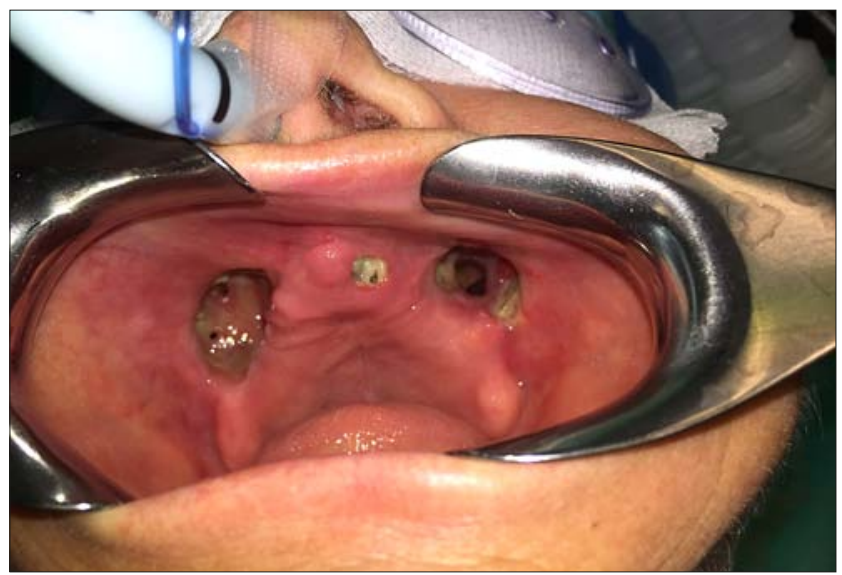

Figure 3. Intraoral view - preoperative osteonecrotic foci

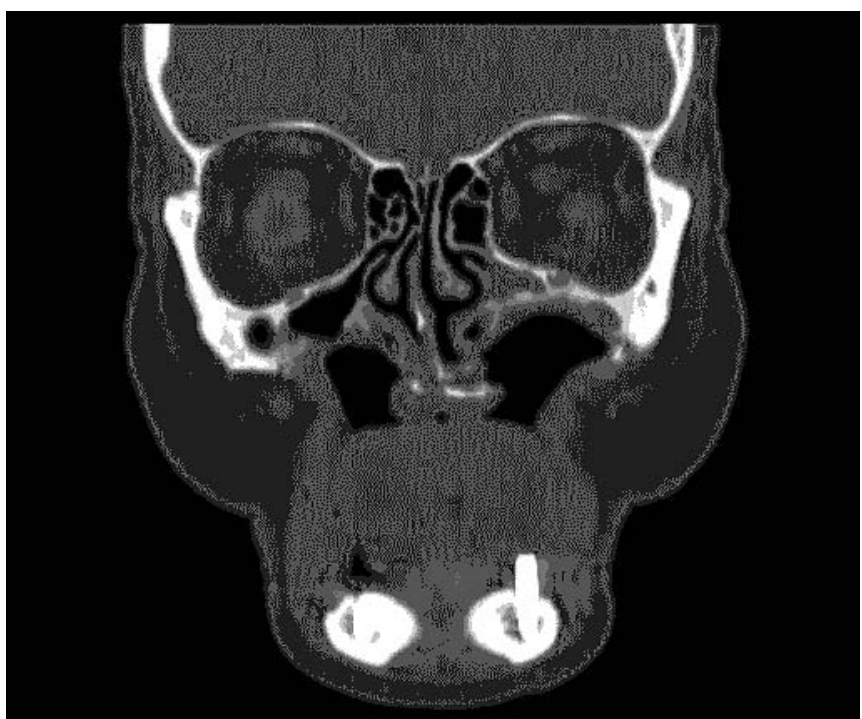

Figure 4. CT scan, complete loss of implants from the jaw, inflammatory process visible on the right side, implants present in position 44,34

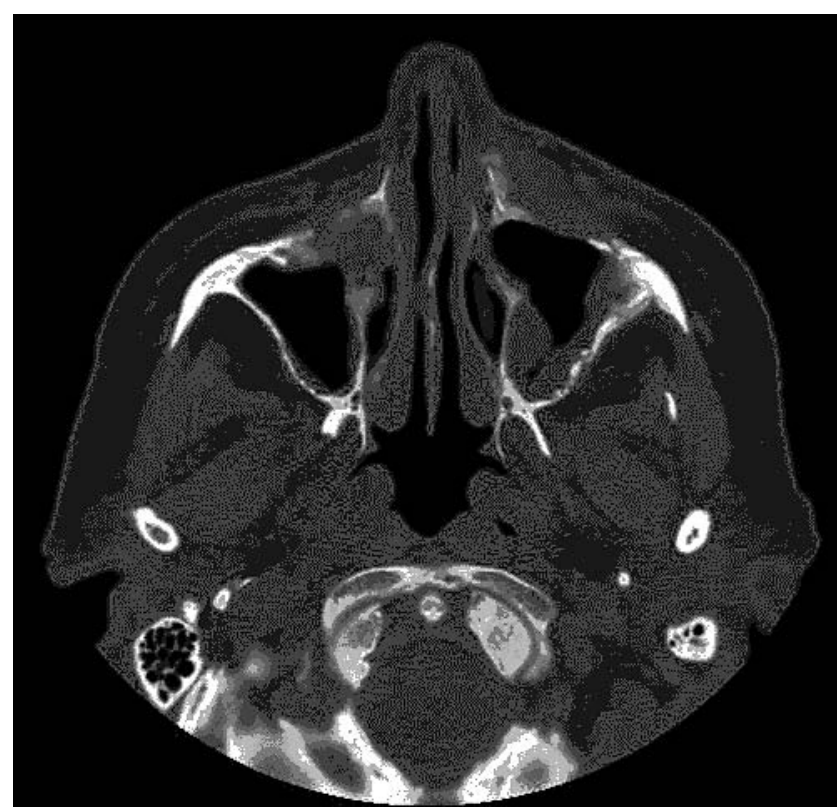

Figure 5. CT scan, visible osteolitic defects of the jaw (anterior and medial wall of the right maxillary sinus and anterior, medial and posterior wall)

Surgical procedure under general anaesthesia included debridement with resection of necrotic parts of the bone and exposed implant 17 in combination with antibiotic therapy. Postoperatively, the patient was administered Klacid $500 \mathrm{mg}$ $2 \mathrm{x} 1$ for 2 weeks. Wounds were left open for second intention healing under sterile gauze and after 2 days under immediate prostheses. Currently, the patient presents healthy mucosa with no signs of inflammation that covered the previously exposed bone. There is no pain nor other symptoms (Fig. 6).

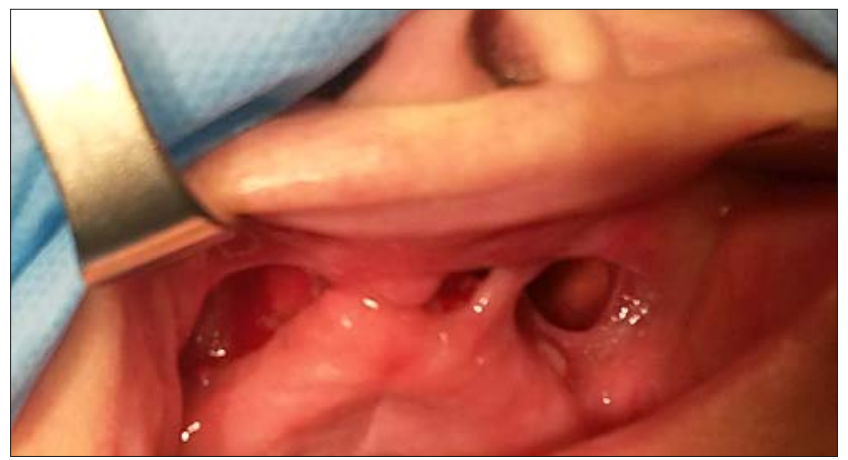

Figure 6. Postoperative view after surgical debridement and resection of necrotic parts of the bone and exposed implant 17. Osteolytic lacunae with healed gingival mucosa and complete epithelial coverage 4 weeks after the operation (black arrows)

\section{DISCUSSION}

Biphosphonates, which are used in various clinical conditions, contribute to necrosis of the jaws and bone exposition in the maxillofacial region $[10,11,3,4,13]$. This disease can be diagnosed relatively easily when a patient presents characteristics such as an exposed bone persisting for more than eight weeks, treatment with antiresorptive or antiangiogenic agents, and no history of radiation therapy to the jaws $[10,11]$. Once the clinical picture shows typical MRONJ, imaging is necessary to determine the size of lesion and a targeted therapy. Exposed bone is not 
always painful, therefore a thorough clinical evaluation and radiological imaging are essential when osteonecrosis is suspected. Preferred imaging options include Panoramic Radiograph (OPG), Cone Bean Computed Tomography (CBCT), Computed Tomography (CT), Magnetic Resonance Imaging (MRI), Nuclear Imaging, Fluorescence-Guided Bone Resection (VELscope). The choice of image modality depends on the surgeon's preference and the available imaging modalities. A three-dimensional imaging modality is desirable and necessary in severe cases, such as for extended resections and planning reconstruction [12]. According to Guo, both OPG and CT could detect the typical symptoms of osseous sclerosis in grade 0 and 1 . For patients of grade 2 , more features were observed on CT. That last modality is also used to analyze the degree of maxillary lesion and soft tissue involvement, compared with OPG [13].

The presented case is that of osteonecrosis of the jaws in a 66-year-old, occupationally-active female who had undergone dental implant treatment, and whose quality of life deteriorated significantly due to the disease. The study shows that MRONJ-like lesions exhibit characteristics such as inflammatory condition around implants in the jaws, mucosal ulceration with exposed necrotic bones and radiopaque alveolar bone. Osteonecrosis of the jaws resulted in the loss of implants, bilateral maxillary sinusotomy and chronic inflammation. Surgical revision and sequestrotomy of the inflammatory foci of the jaw was implemented which led to complete covering of the bone defects with healthy mucosa.

According to Kim et al., it is recommended to delay surgical interventions as long as possible. Despite conservative treatment, surgery may sometimes be necessary due to proceeding necrosis [3] and should be regarded as palliative treatment aimed at deceleration of the progression of the disease, rather than curing the patient and bringing him/ her to health.

In the opinion of Pautke et al., who treated 15 patients with 20 grade II and III osteonecrotic foci of the jaws, mucosal healing of the jaws was achieved in $17 \mathrm{BRONJ}$ patients who did not report any pain [6]. In the presented case, complete mucosal healing was achieved in the patient four weeks after surgical removal of the inflammatory foci of the jaws. In conclusion, the surgical treatment was successful and the patient was occupationally-active again.

Norhold treated 15 patients, among whom 13 presented grade II ONJ lesions and 2 had grade III lesions. The following standardized surgical technique was applied: resection of the necrotic bone, mobilization of the mucoperiosteal flaps and multiple layer coverage of the bone with platelet-rich fibrin (PRF) membranes. On follow-ups, 7 - 10 months after the surgery, complete mucosal healing was achieved in 14 of 15 patients (93\%). The patient with persistent bone exposure had grade III ONJ lesion before the surgery. The use of PRF in the surgical treatment of grade II and III ONJ may be a contributing factor to recovery $[14,15]$.

Prevention and elimination of the risk of MRONJ are highlighted in available literature of the subject. The risk of MRONJ increases along with the bisphosphonates intake which can be controlled in order to improve the quality of life in metastatic patients, and avoid the development of the disease or deceleration or healing of inflammatory lesions [2, $6,3,4]$. Treatment of grade II and III MRONJ is successful not only if the osteonecrotic lesions are completely covered, but also if the development of the disease is stopped without the mucosal healing and symptoms such as pain and odour ex ore subside [6].

Currently, there is no targeted treatment of osteonecrosis due to the lack of comprehensible mechanism of its development. Chinese authors $[3,1,7]$ investigated the therapeutic effect following transplantation of stem cells in miniature pigs. Clinical and radiologic characteristics of BRONJ occurred after extraction of the first premolars with the use of zoledronic acid. This was indicated by decreased Foxp-3 positive regulatory T-cells and an increase in IL-17 in the serum of the pigs treated with BFs. Mucosa healing, as well as reproduction/reconstruction of the osseous tissue, a decrease in the level of IL-17 and increase in the level of Tregs, were observed after allogenic stem cell transplantation. Promising results of in vitro research can be crucial for the treatment of MRONJ [7].

\section{CONCLUSIONS}

- At the beginning of Bisphosphonate-related osteonecrosis of the jaws, conservative therapy supported by targeted antibiotic therapy, thorough oral hygiene and irrigations with the solution of chlorhexidine, are recommended.

- In severe cases of aggravation of inflammatory lesions, surgical treatment is recommended - sequestrotomy of inflammatory foci aimed at inhibition or deceleration of the disease development.

- Change of bisphosphonates administration from intravenous to oral or temporary discontinuation should be considered by the doctor in charge.

\section{REFERENCES}

1. Ogata K, Katagiri W, Osugi M, et al. Evaluation of the therapeutic effects of conditioned media from mesenchymel stem cells in a rat bisphosphonate-related osteoneccrosis of the jaw-like model. Bone 2015; 74: 95-105.

2. Williams WB, O'Ryan F. Management of Medication-related Osteonecrosis of the Jaw Oral and Maxillofacial Surgery Clinics of North America 2015; Volume 27, 4: 517-525.

3. Kim KM, Rhee Y, Kwon T-D, Lee JK, Kim D-Y. Medication Related Osteonecrosis of the jaws: 2015 Position Statement of the Korean Society for bone and mineral research and the Korean Association of oral and maxillofacial surgeons. J Bone Metab. 2015 Nov; 22(4): 151-165.

4. Ruggiero LS.Guidelines for the diagnosis of bisphosphonate-related osteoneccrosis of the jaw (BRONJ). Clin.Cases in Mineral and Bone Metabolism 2007; 4(1): 37-41.

5. Yuxing Guo, Wang D, Wang Y, Peng X and Guo Ch. Imaging features of medicine-related osteonecrosis of the jaws: comparison between panoramic radiography and computed tomography. Oral Surgery, Oral Medicine, Oral Pathology and Oral Radiology 2016; 122, 2: 69-76.

6. Pautke C, Bauer F, Tischer T, Kreutze K, Weit J. Fluorescence-Guided Bone Resection in Bisphosphonate-Associated Osteonecrosis of the Jaws: First clinical results of a prospective pilot study. Journal of Oral and Maxillofacial Surgery 2011; 69: 84-91.

7.AAOMS Updates BRONJ Position Paper: 2014.

8. AAOMS Position Paper on Bisphosphonate-Related Osteonecrosis of the Jaws 2009 Update.

9. Guo Y, Wang D, Wang Y, Peng X at al. Imaging features of medicinerelated osteonecrosis of the jaws: comparison between panoramic radiography and computed tomography. Oral Sur, Oral Med, Oral Path and Oral Radiol, Vol 122, Jan. 2017, e69-e76

10. Li Y, Xu J, Mao L, Liu Y, Gao R, Zheng Z, Chen W, Le A, Shi S, Wang S

11. Allogeneic mesenchymal stem cell therapy for bisphosphonate-related jaw osteonecrosis in Swine. Stem Cells Dev. 2013 Jul 15; 22(14): 2047-56. doi: 10.1089/scd.2012.0615. Epub 2013 Apr. 
12. Lin JH. Biphosphonates: A review of their pharmacokinetic properties. Bone 1996; 18: 75-85.

13. Moreira MS, Katayama E, Bombana AC, Marques MM. Cytotoxity analyses of alendronate on cultured endotelial and subcutaneous tissue. A pilot study. Dent Traumatol. 2005; 21: 329-335.

14. Berg B-J, Mueller AA, Augello M, et al. Imaging in patients with Bisphosphonate-associated osteonecrosis of the jaws (MRONJ). Dent
J. 2016; 4, 3, 29; doi:10.3390/dj4030029 (http://dx.doi.org/103390/ dj4030029)

15. Norholt SE, Hartlev J. Surgical treatment of osteonecrosis of the jaw with the use of platelet-rich fibrin: a prospective study of 15 patients. International Journal of Oral and Maxillofacial Surgery 45, 10, 2016.

16. Burcip Z, Canay G, Asan Y. Treatment of bisphosphonate-related osteonecrosis of the jaw using platelet-rich fibrin CRANIO, The Journal of Craniomandibular and Sleep Practice, 2016. 